Check for updates

Cite this: J. Mater. Chem. C, 2018, 6, 12904

Received 21st September 2018, Accepted 11th November 2018

DOI: $10.1039 / c 8 t c 04780 d$

rsc.li/materials-c

\title{
From colloidal CdSe quantum dots to microscale optically anisotropic supercrystals through bottom-up self-assembly $\dagger$
}

\author{
Elena V. Ushakova, (D)*a Sergei A. Cherevkov, (D) a Dominika-Olga A. Volgina, ${ }^{a}$ \\ Viktor V. Zakharov, ${ }^{a}$ Filipp E. Komissarenko, ${ }^{a}$ Alexey A. Shcherbakov, ${ }^{b}$ \\ Benjamin T. Hogan, iD c Anna Baldycheva, iD c Anatoly V. Fedorov, iD a \\ Igor R. Nabiev (iD de and Alexander V. Baranov (iD)
}

\begin{abstract}
The development of fabrication techniques for novel nanostructured materials is one of the key tasks of modern materials science. One pathway to successfully complete this task is the bottom-up assembly of colloidal nanoparticles into ordered superstructures, possessing both the properties of individual nanoparticles and further novel properties resulting from their interactions. However, nanoparticle self-assembly depends on a variety of parameters, which makes the precise control of this process a complicated problem. Here, the time course of quantum dot (QD) self-assembly into ordered superstructures has been analyzed, along with the evolution of their morphological and optical properties. QD self-assembly occurs through two distinct stages (homo- and hetero-geneous), leading to the formation of supercrystals with a layered morphology. Analysis of the optical properties throughout the superstructures' growth has shown that the absorption and photoluminescence (PL) bands are blue shifted, retaining almost the same PL lifetimes as in the initial QD solution. The supercrystals formed possess a further unique optical property caused by their layered morphology; namely, a four-fold symmetry characterized by strong birefringence. Such supercrystals may be used for the fabrication of microscale optical paths with high extinction coefficients and specific polarization properties for novel optoelectronic devices.
\end{abstract}

\section{Introduction}

Modern technological development requires the design and fabrication of novel materials with parameters fundamentally different from those of any currently available material, such as: small size, novel functionality, high stability, and facile production.

\footnotetext{
${ }^{a}$ ITMO University, 49 Kronverksky pr., Saint Petersburg, 197101, Russia. E-mail: elena.ushakova@corp.ifmo.ru

${ }^{b}$ Moscow Institute of Physics and Technology, 9 Institutsky Lane, Dolgoprudny, Moscow Region, 141701, Russia

${ }^{c}$ University of Exeter, North Park Road, Exeter, UK

${ }^{d}$ Laboratoire de Recherche en Nanosciences, LRN-EA4682, Université de Reims Champagne-Ardenne, 51100 Reims, France

${ }^{e}$ National Research Nuclear University MEPhI "Moscow Engineering Physics Institute", 31 Kashirskoe shosse, 115409 Moscow, Russia

$\dagger$ Electronic supplementary information (ESI) available: Sizes and optical properties of QD samples in solution; microphotographs of the $\mathrm{SC}_{4.0}$ sample of supercrystals; optical images in white light of supercrystals formed by QDs with different diameters; supercrystal morphology calculated by Bragg's law from SAXS pattern; microphotographs of different supercrystals recorded between parallel or crossed polarizers; the optical properties of colloidal solutions of CdSe QDs. See DOI: 10.1039/c8tc04780d
}

These criteria are met perfectly by nanostructured composite materials ${ }^{1}$ based on ordered ensembles of nanoparticles, for example, semiconductor nanocrystal quantum dots (QDs). The properties of these materials are determined by both the intrinsic properties of the nanoparticles included in their structure, and by the novel properties resulting from their interactions. Such superstructured materials are used to manipulate and transform electric and magnetic fields, ${ }^{1,2}$ for obtaining unique optical responses, ${ }^{3}$ generation of coherent light, thermal capacity, and other effects that can be employed in countless applications. As these applications are based on the properties arising from the morphology of the material, the development of protocols for the production of composite materials with controlled morphologies, including strictly determined interparticle distance and mutual orientation of the nanoparticles in the superstructures, is a critical emerging goal. Currently, superstructures can be formed by a number of different methods, which can be divided into top-down (template-assistant, ${ }^{4-6}$ focused ion beam milling, ${ }^{7}$ electron-beam and interference lithography, ${ }^{8}$ and laser beam printing ${ }^{9}$ ) and bottom-up (self-assembly) ${ }^{10}$ processes. Top-down methods of obtaining nanostructured materials 
with a given morphology allow only sequential manipulations with a two-dimensional structure on a solid substrate. Selfassembly, however, makes it possible to obtain three-dimensional structures of a specified shape with a high accuracy, in one step, with a wide range of substrates available, even including flexible substrates. ${ }^{11}$ These composite materials can be used in various areas, including photodetectors, ${ }^{12}$ surface-enhanced Raman spectroscopy sensors, ${ }^{4,5,13}$ light emitters, ${ }^{2}$ and solar cells. ${ }^{14}$ Despite the advantages of this method, it is currently both less controllable and less predictable than "classical" epitaxial approaches. The formation of nanostructured composite materials through selfassembly of nanoparticles is affected by numerous factors, including, amongst others: temperature ${ }^{15,16}$ humidity, ${ }^{15}$ nanoparticle size and concentration in certain solvents, ${ }^{17}$ and ligand type. ${ }^{16,18}$ The development of standardized protocols will enable facile and controllable self-assembly of superstructures, with shapes varying from separate nanoparticulates, ${ }^{19}$ nanoparticle spherical clusters and flower-like structures ${ }^{20}$ to large-scale $2 \mathrm{D}$ - and 3D-superlattices, ${ }^{21-23}$ also called supercrystals (SCs). It should be noted that the properties of such SCs can be controlled both through careful selection of the properties of the individual nanoparticles and modelling of their interactions within the superstructure. ${ }^{24}$ The development of production protocols for materials possessing optical anisotropy is highly attractive in terms of their prospective applications. At present, there are two main approaches to the formation of optically anisotropic materials: (1) synthesis of a structure with an anisotropic morphology, ${ }^{25,26}$ and (2) the use of intrinsically anisotropic nanoparticles as constituent building blocks of a structure. ${ }^{27,28}$ In this paper, we report on the development and study of the morphological and optical properties of the superstructures formed by cadmium selenide QDs. We show that the proposed self-assembly protocol leads to formation of unique SCs with a four-fold symmetry in the visible region of optical spectrum, which has never previously been reported for materials formed by the assembly of isotropic nanoparticles. To reveal the origin of the optical polarization in the material, the changes in the morphology and optical properties of supercrystals with time are traced in parallel, and a model of the optically anisotropic supercrystal formation is proposed.

\section{Results and discussion}

CdSe QDs with diameters of 2.1-4.0 nm have been synthesized by the method described by Reiss et $a .^{29}$ The superstructures are formed through QD self-assembly during slow evaporation of the solvent from a colloidal solution on a substrate. The samples are denoted as $\mathrm{SC}_{\mathrm{dd}}$, where dd denotes the mean QD diameter. For example, $\mathrm{SC}_{4.0}$ denotes a sample with $\mathrm{SCs}$ formed by the self-assembly of $4.0 \mathrm{~nm}$ QDs. Details of the synthesis procedures are presented in the Experimental section.

\subsection{Morphology of supercrystals}

The process of QD supercrystal growth has been investigated by means of optical and electron microscopic examination of an
$\mathrm{SC}_{4.0}$ sample. Analysis of the optical images of the sample showed that the self-organization of the QDs occurred throughout the sample volume and, hence, is strongly dependent on the gradient of the evaporation rate across the sample and the spatial distribution of the QDs. The first SCs began to form within the first $24 \mathrm{~h}$ after sample preparation. All the observed SCs had a planar hexahedral shape with different aspect ratios of the sides (see ESI, $\dagger$ Fig. S1 and S2). Further growth of the SCs took place in one of two ways. At the centre of the drop, a uniform increase in the SC thickness was observed for all SCs, without changes in the lateral dimensions. At the edge of the drop, the growth of a SC occurred along its perimeter only, that is, not at the centre of the crystal. Again, the growth occurred without changes in the lateral dimensions of the initial seed SC. Typical optical images, together with schematic growth patterns for supercrystals, are shown in Fig. 1.

The images in Fig. 1 show that the SC growth occurs stepwise and is thus similar to the formation of common crystals. ${ }^{17,23}$ First, template crystals are formed; in this case, they are planar hexahedra consisting of several QD layers. Then, further growth of the SC occurs, matching the template geometry and symmetry. These two stages of the SCs growing can be described as the homogeneous and heterogeneous stages, ${ }^{30}$ respectively. It should be noted that the character of the second growth stage may vary as a function of the critical QD concentration of the SCsurrounding solvent. ${ }^{17}$ As observed in Fig. 1a and b, if the SC is located in the centre of the QD solution drop, its growth at the heterogeneous stage is uniform over the entire surface of the upper hexahedral plane. However, if there is an insufficient amount of readily available QDs in the droplet close to the SC plane, then QD adsorption will be observed only along the SC perimeter (Fig. 1d and e). The observed layered SC growth is in good agreement with that previously reported by Král et al., ${ }^{31}$ where hexahedral supercrystals were obtained through layer-bylayer growth. To prove the layered morphology of the growing SCs during the growth process, the samples were examined by

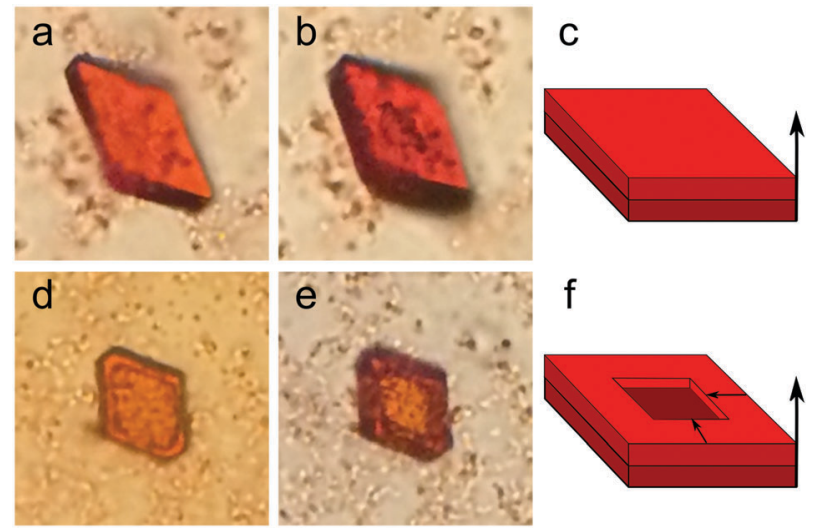

Fig. 1 Optical images of supercrystals appearing in a course of CdSe quantum dots self-assembling. Panels $a, b, d$, and e show optical images $(20 \times 20 \mu \mathrm{m})$ of typical $\mathrm{SC}_{4.0}$ in transmitted white light captured after 12 (a) and 31 (b) days for a layered supercrystal nucleation, and after 3 (d) and 5 (e) days for partly layered supercrystal nucleation. Panels $c$ and $f$ show layered and partly layered supercrystal nucleations, respectively. 


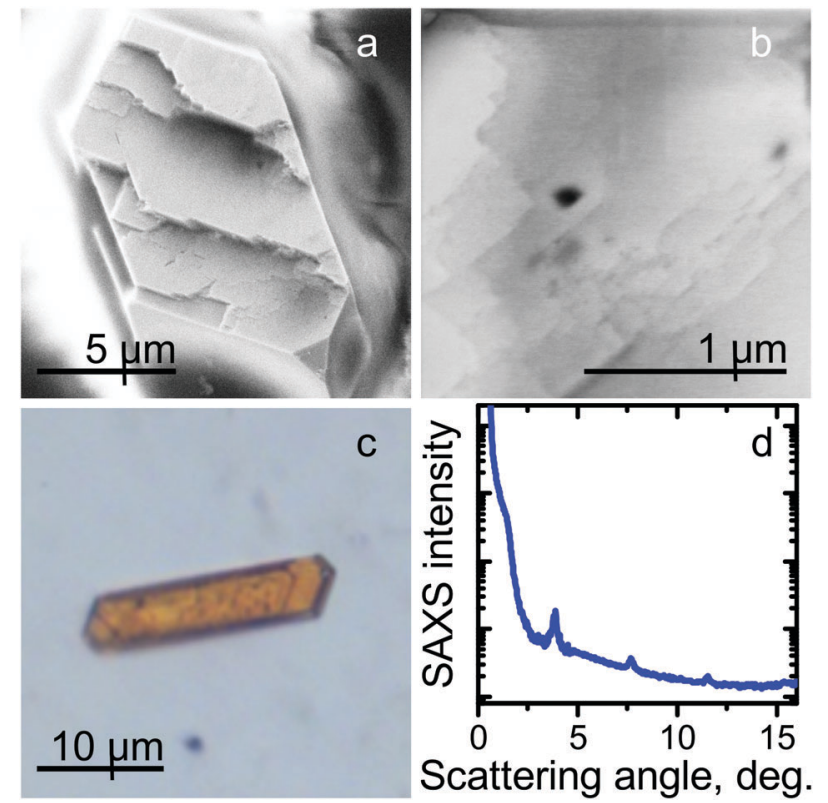

Fig. 2 SEM and optical images and SAXS pattern of the supercrystals. Panels $a$ and $b$ show SEM images of a typical supercrystal. Panel $c$ shows an optical image of a typical supercrystal in reflected light. Panel d show the SAXS pattern of the $\mathrm{SC}_{4.0}$ sample.

means of X-ray scattering and electron microscopy. Fig. 2a and $b$, show typical scanning electron microscope (SEM) images of a SC. The morphological steps corresponding to the layered structure formation are also clearly seen in the optical image in reflected light (Fig. 2c). The small-angle X-ray scattering (SAXS) pattern contains a set of equidistant peaks, as shown in Fig. 2d, which confirms the layered arrangement of the QDs in the SC. The calculated interparticle distance (see details in ESI, $\dagger$ Table S2) is of $4.6 \pm 0.1 \mathrm{~nm}$, which is slightly larger than the QD diameter due to the presence of ligands on the QD surface.

The details of the QD self-assembly process, through the nanoparticles' interactions, leading to formation of a SC may be analyzed according to the approach described by Král et al. ${ }^{31}$ According to this description, the dipole moments of the nanoparticles lie in the horizontal plane, which hence contributes to the layered morphology of the superstructures formed. Furthermore, the formation of layered structures corresponds to the enhanced steric repulsions of the ligands on the QD surface. ${ }^{18}$ As soon as the first QD layer is formed, each "free" QD can then preferentially attach to the existing morphology of the QD dipoles, following the templated pattern. This lies within the framework of the classical nucleation theory, ${ }^{32}$ where nanoparticles are attached to the existing superstructure in a definite crystalline axis/plane, even if it is less energetically favorable during the assembly process. In order to prove the proposed mechanism, we have conducted the same experiment as described above but with a mixture of CdSe QDs of different $(2.1 \mathrm{~nm}$ and $3.1 \mathrm{~nm})$ sizes. A mixture of the two colloidal solutions, with a molar ratio of $1: 1$, was dripped onto a glass substrate. Analysis of micrographs in transmitted light showed that this sample also contained hexahedral SCs (see ESI, $\dagger$ Fig. S2).
However, subsequent investigations of the optical properties of growing superstructures have shown that the formed SCs consisted of $3.1 \mathrm{~nm}$ QDs only. The smaller, $2.1 \mathrm{~nm}$ QDs, did not form ordered structures; instead, they formed disordered aggregates within the droplet. This fact may be explained by the different QD size distributions for the QDs of different sizes. Indeed, the smaller QDs have a larger relative size distribution (see ESI, $\dagger$ Table S1), which limits their ability to self-assemble into highly ordered structures. This observation is in accordance with previous studies, which have shown that the size distribution of the nanoparticles in a colloidal solution is one of the key parameters affecting the assembly of superstructures. ${ }^{21,33}$

\subsection{Optical properties of superstructures}

It has previously been shown that the optical properties of ordered QD ensembles strongly depend on their mutual arrangement within the structure. ${ }^{34,35}$ For instance, the distance between particles may affect the conductive properties of nanoparticle superlattices: the smaller the interparticle distance, the higher the charge carrier mobility. ${ }^{36}$ By assembling anisotropic nanoparticles (nanorods, nanowires, etc.) into low density structures, novel properties such as anisotropy and optical band gaps consequently be observed. ${ }^{37,38}$ In our case, the absorption and PL bands of the SCs are both broadened and shifted towards the blue region of the optical spectrum (i.e. to higher energies) compared to the initial QD colloidal solution. Additionally, analysis of the PL decay showed that the PL lifetimes of QDS in the SCs were almost the same as those observed for the QDs in the colloidal solution, which indicates the absence of additional nonradiative relaxation pathways in the structure. Analysis of the spectral characteristics of QD superstructures by means of confocal microscopy showed that they had a shapedependent distribution. Typical microscopy images of the SCs are shown in Fig. 3.

The data show that the light is intensely absorbed by the entire bulk of the SC (Fig. 3a and b), while the PL signal is observed predominantly from the SC edges (Fig. 3c and d). These unusual properties can be explained by strong interaction of QDs within the SC. For instance, in these samples, appropriate conditions for efficient nonradiative energy transfer may occur, leading to almost fully quenched PL in the SC and, hence, appearance of the PL signal predominantly from the edges of the structure.

Changes in optical properties during qd assembly. To understand how the optical properties of the growing superstructures are connected with the mutual arrangement of the QDs during the assembly process, we tracked the changes of their optical properties with time. The PL parameters of several SCs in a $\mathrm{SC}_{4.0}$ sample were measured over the course of one month. The most distinct changes where observed during the first stage of SC formation: the PL band undergoes a blue shift of $12 \mathrm{~nm}$ and considerable broadening, by $15 \mathrm{~nm}$, compared to the initial QD colloidal solution (Fig. 4a). Further broadening of the PL band was not observed. Fig. $4 \mathrm{~b}$ shows the evolution of the PL peak position over time. A gradual shift of the PL band to the 

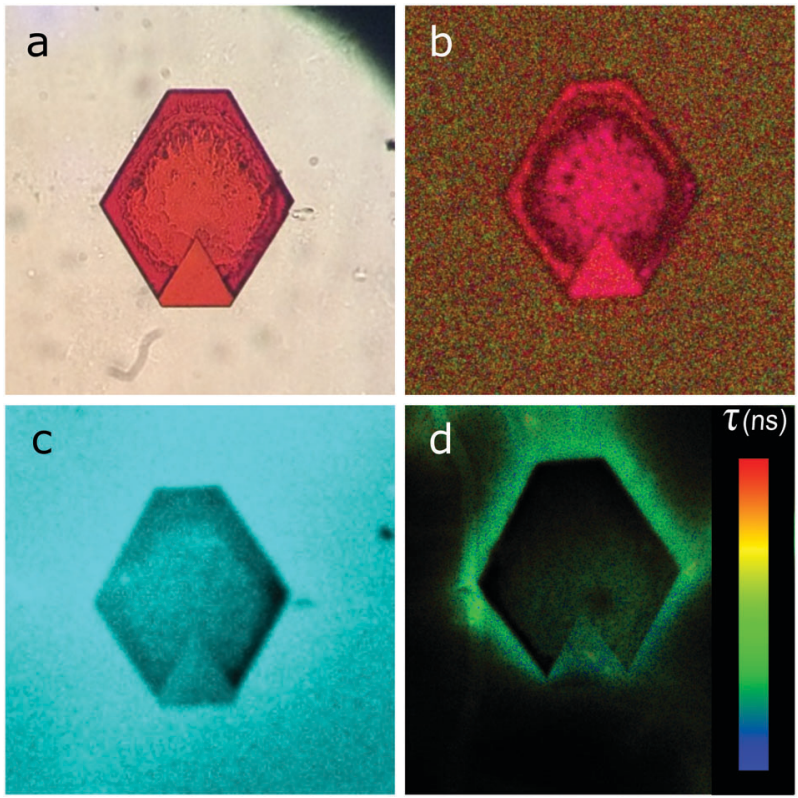

Fig. 3 Optical images of the supercrystals. Panels a-d show microphotographs of supercrystal in transmitted white light (a), a spectral micro-image in white light (b), a luminescence micro-image (c), and a fluorescence lifetime image (FLIM) of the same supercrystal (d). The size of the images is $60 \times 60 \mu \mathrm{m}$.

shorter-wavelength region of the spectrum was observed within the first ten days. The most pronounced changes were detected during the first few hours after sample preparation, as shown in the inset of Fig. 4b. After ten days, the steady-state parameters of the PL remained unchanged. We assume that there is a slight decrease in the size of QD core, similar to observed for the $\mathrm{PbS}$ QD superlattices, ${ }^{39}$ accompanied with a change in the dielectric permittivity of the surrounding media compared to the QD solution, which may result in the blue shift of the QD PL band. The observed trend in the PL agrees with the results of morphological studies of the SCs. During the first $24 \mathrm{~h}$, at the stage of homogeneous nucleation, small flat crystals were formed, which correlates with the greatest changes in the PL parameters. Then, at the heterogeneous stage of crystal growth, the PL parameters were observed to be changing more gradually.

While the QDs were assembling, an unexpected increase in the average PL lifetime was observed. Fig. 4c and $\mathrm{d}$ shows typical PL decay and average lifetime curves. At the first stage of QDs assembly, the PL lifetime decreased from $35 \mathrm{~ns}$, which corresponded to that of the QD colloidal solution, to $21 \mathrm{~ns}$. Then, the average PL lifetime slowly increased and reached values typical of the colloidal QD solution. We believe that this change was due to the reduction of the number of nonradiative relaxation channels during the QDs' self-assembly into an ordered structure. The concentration of the oleic acid in the QD SCs is increased compared to that in the QD solution, which was examined by FTIR spectra. The increase in the ligand concentration in the vicinity of QDs leads to better QD surface passivation, and hence, to an increase of the quantum yield, as was shown in ref. 40. In our case, the formation of such
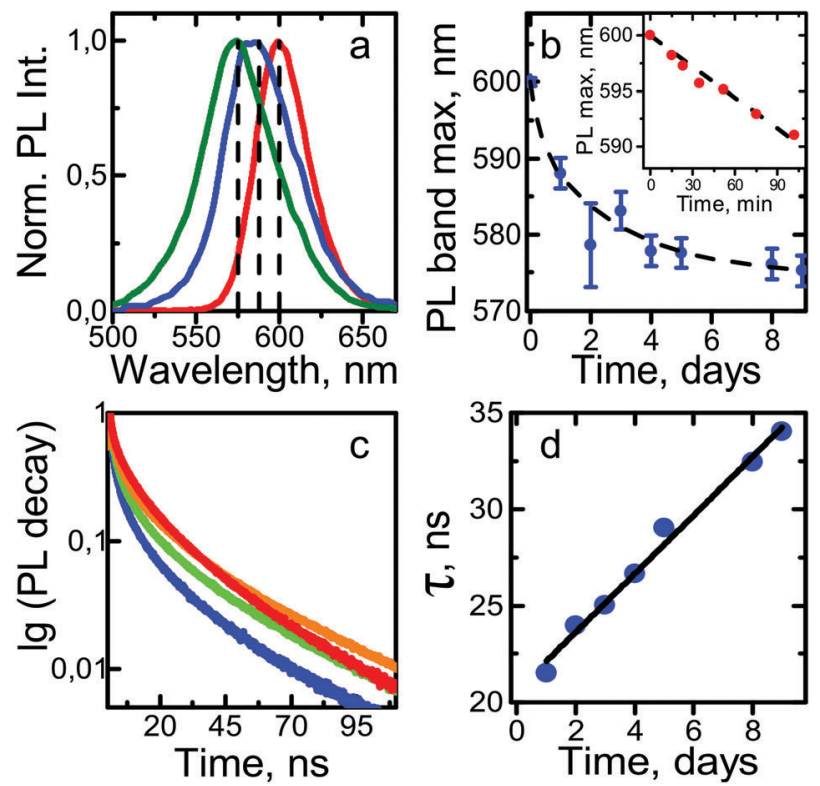

Fig. 4 Time course of the variation of the superstructures' optical properties during QDs self-assembly. Panel a shows the PL spectra obtained one (blue) and nine (green) days after sample preparation, compared with the PL spectra for the initial QD solution (red curve). Panel b shows changes in the PL peak position as a function of the time; the inset shows the changes within the first two hours of SC growth. Panel c shows the PL decay curves obtained after 1 (blue), 4 (green), and 25 (orange) days compared to the initial QD solution (red curve). Panel d shows changes in the average PL lifetime as a function of SC growthtime. The dashed lines in the plots are guides for the eye.

superstructures from QDs with increased ligand concentration allows one to maintain the same average PL lifetime as in the colloidal solution. It is worth mentioning that the decay of the QD PL in the solution fitted a biexponential function (shown in Fig. $4 \mathrm{c}$ as a red line), in contrast to a triexponential function in the case of the assembled QDs.

Optical anisotropy of the supercrystals. We have further examined the polarization properties of the SCs in transmitted and reflected light respectively. The transmitted light intensity depends on the angle of rotation of the SC between crossed polarizers. Fig. 5 shows microphotographs of the SCs obtained in transmitted (a-d) and reflected (e and f) light, and the angular dependences of transmission and reflection for typical supercrystals from the $\mathrm{SC}_{3.1}$ and $\mathrm{SC}_{4.0}$ samples. It is worth mentioning that the same behavior is observed while using objectives with different numerical apertures (NA) and also changing the size of the diaphragm on the condenser to obtain a beam with close to parallel light propagation. More microphotographs of different SCs between crossed polarizers are presented in the ESI, $\dagger$ Fig. S3.

The maximum transmitted light intensity was observed when the longest edge of an SC was inclined 30 degrees to the polarizer axis. Fig. 5 h shows that the angular dependence in reflected light had the same shape and symmetry as in transmitted light. In this case, the angle of the maximum reflection signal in crossed polarizers relative to the orientation of the 

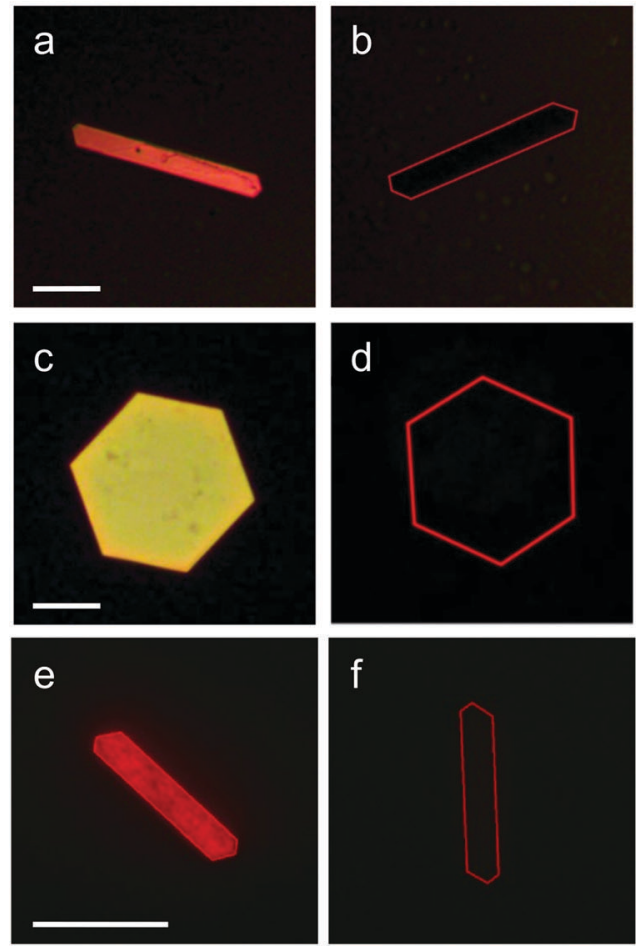
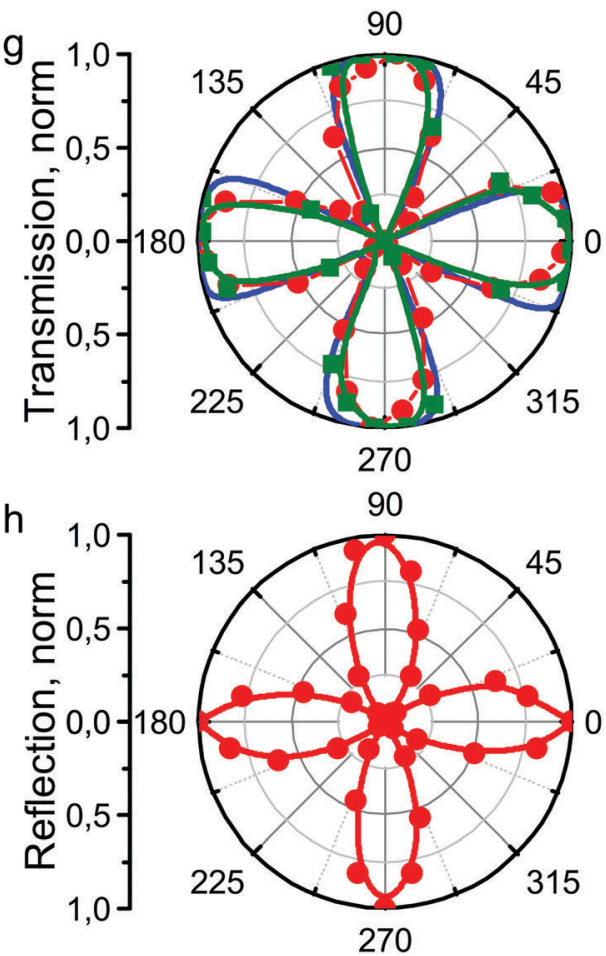

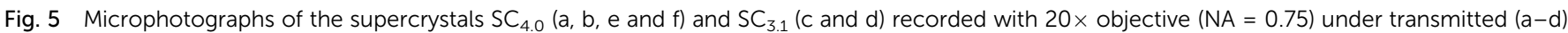

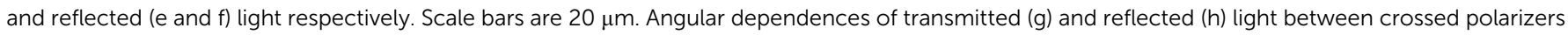
for $\mathrm{SC}_{4.0}$ (red curves), $\mathrm{SC}_{3.1}$ (green curve), and the organic matrix (blue curve).

longest SC edge was also 30 degrees. In order to understand how the optical parameters of the QDs change with sample rotation, we conducted a spectral study of the light transmitted through the SCs. Fig. 6 shows that the form of the transmission spectrum did not change as the sample was rotated, whereas the amplitude of the transmitted light varied. After that, the PL signal in crossed polarizers was examined and found to be independent of the angular position of the SC sample relative to the polarizer axis.

Hence, the optical effects observed in transmitted and reflected light between crossed polarisers were not related to the mutual arrangement of QDs within the SC. We therefore

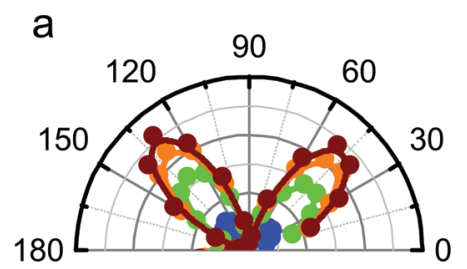

Transmission at wavelength, $\mathrm{nm}$ :

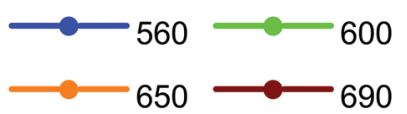

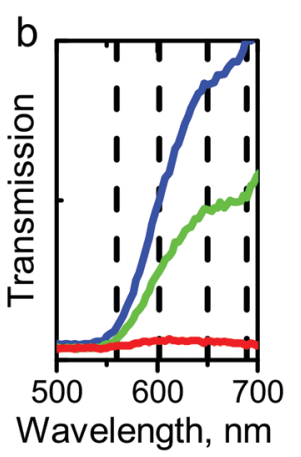

Fig. 6 Angular dependences of transmitted light at different wavelengths (a), shown in (b) as dashed lines. Panel b shows transmission spectra of a typical supercrystal measured at the angles of 50 (blue), 70 (green) and 90 (red) degrees. assume that the chemical environment of the QDs in the SC affected the polarization properties of the sample. In order to confirm this assumption, the polarization properties of a lyotropic crystal sample formed by a three-phase system consisting of oleic acid, water, and tetrachloromethane were investigated. The angular dependence of the transmitted light for the lyotropic crystal was found to be the same as for QD supercrystals (Fig. 5g, blue curve). The angular dependences of the transmittance and reflectance indicate that the SCs possess birefringence. Let us therefore consider this phenomenon in detail.

In Fig. 7a, the polarization directions of two eigenwaves in the crystal are denoted $a_{1}$ and $a_{2}$. The direction of light propagation is orthogonal to the plane of the figure. Let us assume that the directions of the polarizer and the analyser, $a_{1}$ and $a_{2}$, are rotated by angle $\alpha$ relative to $b_{1}$ and $b_{2}$, respectively. Then, taking the incident wave amplitude to be $E_{0}$, one first determines the amplitudes of the two waves propagating in the crystal at different velocities: $E_{0} \cdot \cos (\alpha)$ and $E_{0} \cdot \sin (\alpha)$. Upon exiting the crystal, these waves should be projected onto the analyser direction $b_{2}$. Additionally, they should gain a phase difference $\phi$ depending on the crystal thickness. Thus, the amplitude measured by the analyser can be expressed as $E=E_{0} \cdot \sin (\alpha) \cdot \cos (\alpha) \cdot(1-$ $\left.\exp ^{i \phi}\right)$. If the crystal thickness changes across the beam, the resulting measured power can be averaged over the phase $\phi$ : $P=\int E_{0}^{2} \cdot \sin ^{2}(\alpha) \cdot \cos ^{2}(\alpha) \cdot\left|\left(1-\exp ^{i \phi}\right)\right|=E_{0}^{2} \cdot \sin ^{2}(\alpha) \cdot \cos ^{2}(\alpha)$.

The $P(\alpha)$ function plot is presented in Fig. 7b. The results of modeling agree with the dependences obtained for the 
a

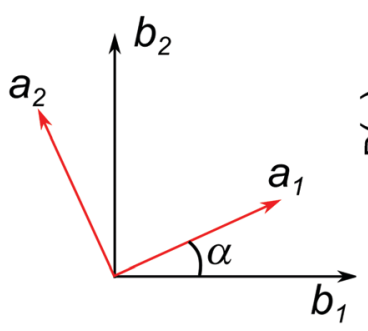

b

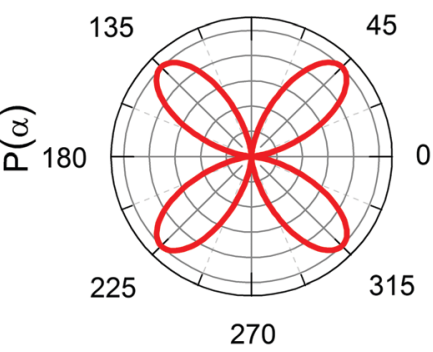

Fig. 7 A scheme of the crystal axes (red) and polarizer and analyser directions (black) are shown in panel $a$; the $P(\alpha)$ function is presented in panel b.

supercrystals. It is well known that crystals whose crystal lattice has a six-fold symmetry, with the main optical axis perpendicular to the polarizer plane, should not possess optical anisotropy. ${ }^{41}$ However, as our experiments show the opposite, the observed polarization properties should then be determined by further effects perturbing a six-fold symmetry of the SC. Three possible explanations may be proposed.

(1) If we consider QD supercrystals as a structure with alternating layers of 2D QD ensembles and oleic acid molecules, we can assume that a form birefringence can be achieved, as was shown in related literature. ${ }^{42-45}$ In this case, the effective refractive indices of the light propagation may be changed resulting in the anisotropic behavior of the light transmitted through the structure even in the plane of normal incidence. This observation also correlates with the polarization properties of the lyotropic crystals formed by surfactant molecules, ${ }^{46-48}$ which is similar to our observations of the anisotropy of the test sample (organic matrix) formed by a three-phase oleic acid/nonpolar solvent/water system.

(2) The inner morphology of SCs possesses a crystal symmetry group different from hexagonal. In that case, at least one optical axis should not be perpendicular to the observation $X Y$-plane in the experiment, thus forming an inclined optical axis in the supercrystal. The QD superlattices can also form different polymorphs of approximately similar symmetry groups. ${ }^{49}$ Thus, the observed SC shape, in our case, can also be attributed to the tetragonal crystal lattice, and more specifically for the case of the close-packed nanoparticles - the face-centered tetragonal structure.

(3) The SCs actually possess hexagonal, tetragonal or even cubic symmetry (face- or body-centered), but inner stress in the distorted crystal lattice causes optical anisotropy. However, further experiments are needed to fully develop and check these hypotheses.

\section{Conclusions}

We have demonstrated the development of, and conducted studies on the morphological and optical properties of the superstructures formed by the bottom-up self-assembly of cadmium selenide QDs. At present, there are two main approaches to the formation of optically anisotropic materials, which include synthesis of a structure with an anisotropic morphology or the use of intrinsically anisotropic nanoparticles as building blocks. We have demonstrated that our proposed self-assembly protocol lead to formation of uniquely birefringent supercrystals with a four-fold symmetry in the visible region of the optical spectrum, which has never previously been reported for materials formed by isotropic nanoparticles. In order to reveal the origin of the optical polarization in such material, the changes in the morphology and optical properties of supercrystals with time were traced in parallel, and a model of the optically anisotropic supercrystal formation was proposed. Developed supercrystals may be used for fabrication of microscale optical paths with high extinction coefficients and specific polarization properties for novel optoelectronic devices.

\section{Experimental section}

\section{Experimental setup}

For steady-state measurements of optical properties, an UV3600 spectrophotometer (Shimadzu, Japan), and a Cary Eclipse spectrofluorimeter (Aligent, USA), were used. To investigate the PL kinetics of the SC samples at room temperature, a MicroTime 100 device (PicoQuant, Germany) employing time-correlated single-photon counting, equipped with a $100 \times$ objective lens $(\mathrm{NA}=0.95)$ and a picosecond pulsed diode laser head $(\lambda=405 \mathrm{~nm})$, was used. PL decay curves were fitted by a multiexponential function: $I(t)=I_{0}+\sum_{i} A_{i} \tau_{i}$. The average PL lifetime has been calculated as $\tau_{\mathrm{av}}=\frac{\sum_{i} A_{i} \tau_{i}^{2}}{\sum_{i} A_{i} \tau_{i}}$. To obtain the luminescence images of the samples along with the investigation of the time course PL spectral parameters of typical supercrystals during self-assembly, we used an LSM 710 confocal laser scanning microscope (Carl Zeiss, Germany) built on the basis of an Axio Imager Z1 direct fluorescence microscope (Carl Zeiss, Germany). To measure the absorption spectra by means of a confocal microscope, a special mode of operation was used, in which the excitation source was activated through special macros. ${ }^{50}$ As a source of illumination, a halogen incandescent lamp was used, the light from which was focused on the sample by means of a condenser. The signal passing through the sample was collected by a lens with a large aperture and then recorded by a spectral photodetector. The measurements were carried out by a comparative method; light transmitted through a glass slide was used as a comparison sample. Polarization measurements were carried out using crossed polarizers, in the spectral mode of the confocal microscope, equipped with a set of objectives with varied NA $(0.2-0.95)$. The sample was placed on a rotating table. The aperture diaphragm of the condenser was kept as small as possible for each individual measurement. The test experiment with an isotropic glass slide did not reveal any change in the transmitted signal while rotating the sample in this optical scheme.

\section{Sample preparation}

Cadmium selenide QDs were synthesized by the hot-injection method, in accordance with the protocol described by Reiss et al. ${ }^{29}$ 
and stabilized by trioctylphosphine oxide (TOPO). The diameters of the QDs synthesized were $2.1 \mathrm{~nm}, 3.1 \mathrm{~nm}$, and $4.0 \mathrm{~nm}$ in different samples. The positions of ABS and PL bands together with the mean QD size and its distribution are listed in ESI, $\dagger$ Table S1. For subsequent QD assembly, TOPO ligands at the QD surface were replaced with oleic acid molecules, and the QDs were redispersed in tetrachloromethane (TCM). The absorption and PL spectra are shown in ESI, $\dagger$ Fig. S4. Superstructures were formed by dripping a $10^{-7} \mathrm{M}$ colloidal QD solution onto a glass substrate. The QD assembly lasted from one day to one week. During the assembly process the SC samples were measured and stored under the atmosphere at ambient conditions. The optical properties, including anisotropy, of at least 20 supercrystals were examined.

\section{Author contributions}

E. U., D. V., V. Z. conducted the optical experiments. S. C. synthesized QDs. F. K., B. T. and An. B. characterized the morphology of the samples. A. S. proposed a model of optical anisotropy. E. U., B. T. and I. N. wrote the paper. A. F., I. N. and Al. B. supervised the project.

\section{Conflicts of interest}

There are no conflicts of interest to declare.

\section{Acknowledgements}

Authors thank Dr I. A. Kasatkin for SAXS measurement at Saint Petersburg State University. This study was supported by the Ministry of Education and Science of the Russian Federation through the grant No. 14.584.21.0032 (ID RFMEFI58417X0032), the Engineering and Physical Sciences Research Council (EPSRC) of the United Kingdom via the EPSRC Centre for Doctoral Training in Electromagnetic Metamaterials (Grant No. EP/L015331/1) and via EP/N035569/1, and the Royal Society via International Exchange Grant No. 2016/R1.

\section{References}

1 X. C. Tong, Functional Metamaterials and Metadevices, Springer, 2018.

2 A. A. Kulkarni, J. Kohanek, K. I. Tyler, E. Hanson, D.-U. Kim, K. Thornton and P. V. Braun, Adv. Opt. Mater., 2018, 6, 1800071.

3 I.-Y. Chang, D. Kim and K. Hyeon-Deuk, ACS Appl. Mater. Interfaces, 2017, 9, 32080-32088.

4 C. Tian, Y. Deng, D. Zhao and J. Fang, Adv. Opt. Mater., 2015, 3, 404-411.

5 D. S. Kim, A. Honglawan, S. Yang and D. K. Yoon, ACS Appl. Mater. Interfaces, 2017, 9, 7787-7792.

6 C. Hanske, G. González-Rubio, C. Hamon, P. Formentn, E. Modin, A. Chuvilin, A. Guerrero-Martnez, L. F. Marsal and L. M. Liz-Marzán, J. Phys. Chem. C, 2017, 121, 10899-10906.
7 M. C. Dalfovo, L. J. Giovanetti, J. M. Ramallo-López, R. C. Salvarezza, F. G. Requejo and F. J. Ibañez, J. Phys. Chem. C, 2015, 119, 5098-5106.

8 M. S. Saifullah, M. Asbahi, M. Binti-Kamran Kiyani, S. Tripathy, E. A. Ong, A. Ibn Saifullah, H. R. Tan, T. Dutta, R. Ganesan and S. Valiyaveettil, et al., ACS Nano, 2017, 11, 9920-9929.

9 E. Tiguntseva, G. P. Zograf, F. E. Komissarenko, D. A. Zuev, A. A. Zakhidov, S. V. Makarov and Y. S. Kivshar, Nano Lett., 2018, 18, 1185-1190.

10 R. Tan, H. Zhu, C. Cao and O. Chen, Nanoscale, 2016, 8, 9944-9961.

11 Y. Wang, M. Li, E. Colusso, W. Li and F. G. Omenetto, Adv. Opt. Mater., 2018, 6, 1800066.

12 A. Maulu, P. J. Rodrguez-Cantó, J. Navarro-Arenas, R. Abargues, J. F. Sánchez-Royo, R. Garca-Calzada and J. P. M. Pastor, RSC Adv., 2016, 6, 80201-80212.

13 L. Scarabelli, C. Hamon and L. M. Liz-Marzán, Chem. Mater., 2016, 29, 15-25.

14 S. M. Rupich, Y. N. Gartstein, A. V. Malko and Y. J. Chabal, Adv. Opt. Mater., 2016, 4, 378-383.

15 S. Gómez-Graña, J. Pérez-Juste, R. A. Alvarez-Puebla, A. Guerrero-Martnez and L. M. Liz-Marzán, Adv. Opt. Mater., 2013, 1, 477-481.

16 M. A. Cordeiro, E. R. Leite and E. A. Stach, Langmuir, 2016, 32, 11606-11614.

17 Z. Wang, K. Bian, Y. Nagaoka, H. Fan and Y. C. Cao, J. Am. Chem. Soc., 2017, 139, 14476-14482.

18 D. Gentili, G. Ori, L. Ortolani, V. Morandi and M. Cavallini, ChemNanoMat, 2017, 3, 874-878.

19 X. Wang, L. Lin, R. Liu, M. Chen, B. Chen, B. He, B. He, X. Liang, W. Dai and H. Zhang, et al., Adv. Funct. Mater., 2017, 27, 1700406.

20 T. Kormilina, E. Stepanidenko, S. Cherevkov, A. Dubavik, M. Baranov, A. Fedorov, A. Baranov, Y. Gun'ko and E. Ushakova, J. Mater. Chem. C, 2018, 6, 5278-5285.

21 E. V. Ushakova, S. A. Cherevkov, A. P. Litvin, P. S. Parfenov, I. A. Kasatkin, A. V. Fedorov, Y. K. Gun'ko and A. V. Baranov, Nanoscale, 2018, 10, 8313-8319.

22 C.-Y. Chiu, C.-K. Chen, C.-W. Chang, U.-S. Jeng, C.-S. Tan, C.-W. Yang, L.-J. Chen, T.-J. Yen and M. H. Huang, J. Am. Chem. Soc., 2015, 137, 2265-2275.

23 M. A. Boles and D. V. Talapin, J. Am. Chem. Soc., 2015, 137, 4494-4502.

24 N. Kholmicheva, P. Moroz, H. Eckard, G. Jensen and M. Zamkov, ACS Energy Lett., 2016, 2, 154-160.

25 J.-B. Wu, H. Zhao, Y. Li, D. Ohlberg, W. Shi, W. Wu, H. Wang and P.-H. Tan, Adv. Opt. Mater., 2016, 4, 756-762.

26 A. A. Ushkov and A. A. Shcherbakov, Opt. Express, 2017, 25, 243-249.

27 S. Sucharitakul, G. Ye, W. R. Lambrecht, C. Bhandari, A. Gross, R. He, H. Poelman and X. P. Gao, ACS Appl. Mater. Interfaces, 2017, 9, 23949-23956.

28 A. Singh, A. Singh, G. K. Ong, M. R. Jones, D. Nordlund, K. Bustillo, J. Ciston, A. P. Alivisatos and D. J. Milliron, Nano Lett., 2017, 17, 3421-3428. 
29 M. Protière, N. Nerambourg, O. Renard and P. Reiss, Nanoscale Res. Lett., 2011, 6, 472.

30 M. A. Boles, M. Engel and D. V. Talapin, Chem. Rev., 2016, 116, 11220-11289.

31 D. V. Talapin, E. V. Shevchenko, C. B. Murray, A. V. Titov and P. Král, Nano Lett., 2007, 7, 1213-1219.

32 F. Abraham, Homogeneous nucleation theory, Academic Press, New York, 1974, vol. 1.

33 C. Wang, C. Siu, J. Zhang and J. Fang, Nano Res., 2015, 8, 2445-2466.

34 E. V. Ushakova, S. A. Cherevkov, A. P. Litvin, P. S. Parfenov, V. V. Zakharov, A. Dubavik, A. V. Fedorov and A. V. Baranov, Opt. Express, 2016, 24, A58-A64.

35 A. S. Baimuratov, Y. K. Gun'ko, A. V. Baranov, A. V. Fedorov and I. D. Rukhlenko, Sci. Rep., 2016, 6, 23321.

36 J. Huang, X. Wang, N. L. Hogan, S. Wu, P. Lu, Z. Fan, Y. Dai, B. Zeng, R. Starko-Bowes and J. Jian, et al., Adv. Sci., 2018, 1800416.

37 X. Bouju, É. Duguet, F. Gauffre, C. R. Henry, M. L. Kahn, P. Mélinon and S. Ravaine, Adv. Mater., 2018, 1706558.

38 S. Zhang, C. I. Pelligra, X. Feng and C. O. Osuji, Adv. Mater., 2018, 30, 1705794.

39 E. V. Ushakova, S. A. Cherevkov, A. P. Litvin, P. S. Parfenov, D.-O. A. Volgina, I. A. Kasatkin, A. V. Fedorov and A. V. Baranov, J. Phys. Chem. C, 2016, 120, 25061-25067.
40 M. A. Boles, D. Ling, T. Hyeon and D. V. Talapin, Nat. Mater., 2016, 15, 141.

41 F. S. Crawford, Waves, Berkeley physics course, 1968.

42 Y. G. Boucher, J. Le Rouzo, I. Ribet-Mohamed and R. Hadar, J. Opt. Soc. Am. B, 2008, 25, 777-784.

43 M. D. Robinson, G. Sharp and J. Chen, Polarization engineering for LCD projection, John Wiley \& Sons, 2005, vol. 4, pp. $47-75$.

44 A. Waddie, R. Buczynski, F. Hudelist, J. Nowosielski, D. Pysz, R. Stepien and M. Taghizadeh, Opt. Mater. Express, 2011, 1, 1251-1261.

45 H. Yang, H. Jussila, A. Autere, H.-P. Komsa, G. Ye, X. Chen, T. Hasan and Z. Sun, ACS Photonics, 2017, 4, 3023-3030.

46 L. Coppola, R. Gianferri, I. Nicotera, C. Oliviero and G. A. Ranieri, Phys. Chem. Chem. Phys., 2004, 6, 2364-2372.

47 R. Ranjini, M. V. Matham and N.-T. Nguyen, Opt. Mater., 2011, 33, 1338-1341.

48 J. J. Vallooran, S. Handschin, S. M. Pillai, B. N. Vetter, S. Rusch, H.-P. Beck and R. Mezzenga, Adv. Funct. Mater., 2016, 26, 181-190.

49 Z. Wang, C. Schliehe, K. Bian, D. Dale, W. A. Bassett, T. Hanrath, C. Klinke and H. Weller, Nano Lett., 2013, 13, 1303-1311.

50 V. Zakharov, M. Baranov, A. Zlatov and A. Veniaminov, Nanosyst.: Phys., Chem., Math., 2014, 5, 811-819. 